OPEN

SUBJECT AREAS:

DISEASE GENETICS

GENETICS RESEARCH

Received

17 March 2014

Accepted

18 August 2014

Published

9 October 2014

Correspondence and requests for materials should be addressed to M.K. (marcin. krawczyk@uks.eu)

* These authors contributed equally to this work.

\section{Variant adiponutrin confers genetic protection against cholestatic itch}

\author{
Marcin Krawczyk', Malgorzata Milkiewicz ${ }^{2}$, Hanns-Ulrich Marschall ${ }^{3}$, Clemens Bartz ${ }^{4}$, Frank Grünhage' , \\ Ewa Wunsch ${ }^{5}$, Piotr Milkiewicz ${ }^{5,6 *} \&$ Frank Lammert $^{1 *}$
}

\begin{abstract}
${ }^{1}$ Department of Medicine II, Saarland University Medical Center, Saarland University, Homburg, Germany, ${ }^{2}$ Medical Biology Laboratory, Pomeranian Medical University, Szczecin, Poland, ${ }^{3}$ Sahlgrenska Academy, Institute of Medicine, Department of Molecular and Clinical Medicine, University of Gothenburg, Gothenburg, Sweden, ${ }^{4}$ Department of Gynecology and Obstetrics, Klinikum Saarbrücken, Saarbrücken, Germany, ${ }^{5}$ Liver Research Laboratories, Pomeranian Medical University, Szczecin, Poland, 'Liver and Internal Medicine Unit, Department of General, Transplant and Liver Surgery, Medical University of Warsaw, Warsaw, Poland.
\end{abstract}

Lysophosphatidic acid (LPA) mediates cholestatic pruritus. Recently the enzyme PNPLA3, expressed in liver and skin, was demonstrated to metabolise LPA. Here we assess the association of the PNPLA3 variant p.Ile148Met, known to be associated with (non-)alcoholic fatty liver disease (NAFLD) in genome-wide association studies, with cholestatic itch in 187 patients with primary biliary cirrhosis (PBC) and 250 PBC-free controls as well as 201 women with intrahepatic cholestasis of pregnancy (ICP) and 198 female controls without a history of ICP. Our hypothesis was that the intensity of cholestatic itch differs in carriers of distinct PNPLA3 p.Ile148Met genotypes. Patients with PBC carrying the allele p.148Met that confers an increased NAFLD risk reported less itching than carriers of the p.148Ile allele (ANOVA $P=0.048$ ). The $P N P L A 3$ p.148Ile allele increased the odds of requiring plasmapheresis for refractory pruritus $(\mathrm{OR}=3.94$, 95\% CI $=0.91-17.00, \mathrm{P}=0.048$ ). In line with these findings, the PNPLA3 p.148Met allele was underrepresented in the ICP cohort $(\mathrm{OR}=0.66,95 \% \mathrm{CI}=0.47-0.92, \mathrm{P}=0.013)$. Notwithstanding the need for further replication of these findings, we conclude that the PNPLA3 allele p.148Met might confer protection against cholestatic pruritus, possibly due to increased LPA-acyltransferase activity in liver and/or skin.

ecent studies established the adiponutrin (PNPLA3) variant p.Ile148Met as common genetic risk factor for severe forms of chronic liver diseases ${ }^{1}$. Indeed, patients with non-alcoholic ${ }^{2,3}$ and alcoholic fatty liver disease ${ }^{4,5}$ who carry the p.148Met allele at this locus are prone to progressive liver fibrosis and cirrhosis. In our elastography-based analysis ${ }^{6}$, we demonstrated that this allele is associated with increased liver fibrosis in patients with chronic liver diseases in general. PNPLA3 has been reported to be a triacylglycerol hydrolase or lysophosphatidic acid (LPA) acyltransferase, and the p.Ile148Met variant might affect the remodelling of lipids, including the conversion of LPA into phosphatidic $\operatorname{acid}^{1,7}$. Carriers of the p.148Met allele might have have increased LPA catabolism, which promotes the synthesis of diacylglycerol and modulates the composition of lipid droplets.

To date, the treatment of pruritus in patients with liver diseases remains a troublesome challenge. Although several drugs (ursodeoxycholic acid, rifampicin) and invasive approaches (nasobiliary drainage, plasmapheresis) represent therapeutic options $s^{8}$, they are not effective in many patients. Clinical observations support the notion that patients with comparable grade of cholestasis display different severity of pruritus'. Thus genetic predisposition might affect the degree of itching, but to date no common pruritus-related genes that are associated with treatment-refractory pruritus have been identified. In addition to liver, skin also shows high PNPLA3 expression levels ${ }^{10}$. Interestingly, LPA has been identified as the critical mediator of cholestatic pruritus ${ }^{11}$. Indeed, LPA binds to the $\mathrm{LPA}_{1}$ receptor in skin and causes itching ${ }^{11}$. Patients with cholestatic pruritus are characterized by increased serum concentrations of LPA, which can be generated from lysosphatidylcholine by the enzyme autotoxin in blood ${ }^{11}$. Therefore we hypothesized that the polymorphism p.Ile148Met of PNPLA3, which catabolizes LPA, affects the severity of cholestatic pruritus and investigated two independent cohorts of patients with cholestatic liver diseases (Tables 1 and 2).

\section{Results}

As shown in Figure 1 and Supplementary Table 1, the intensity of itch differed significantly between carriers of different genotypes of the PNPLA3 variant p.Ile148Met (ANOVA P $=0.048$ ). Moreover, the PNPLA3 allele 
Table 1 | Demographic, clinical and laboratory data in PBC patients and controls

\begin{tabular}{lcc} 
& $\mathrm{PBC}(\mathrm{n}=187)$ & Controls $(\mathrm{n}=250)$ \\
\hline Age (years) & $56(22-83)$ & $25(18-66)$ \\
Gender (women/men) & $166 / 21$ & $210 / 40$ \\
Liver cirrhosis (yes/no/ & $69 / 115 / 3$ & $0 / 250 / 0$ \\
$\quad$ Unknown) & $162 / 25$ & $\mathrm{ND}$ \\
AMA (positive/negative) & $85(10-987)$ & $\mathrm{ND}$ \\
ALT (IU/I) & $323(37-1899)$ & $\mathrm{ND}$ \\
AP (IU/I) & $295(11-1932)$ & $\mathrm{ND}$ \\
$\boldsymbol{\gamma}$-GT (IU/I) & $3.2(0.2-45.5)$ & $\mathrm{ND}$ \\
Bilirubin (mg/dl) & &
\end{tabular}

Values are given as medians (ranges), unless stated otherwise.

Abbreviations: ALT, alanine aminotransferase; AMA antimitochondrial antibodies; $\mathrm{AP}$, alkaline phosphatase; $\gamma$-GT, $\gamma$-glutamyl transpeptidase; ND, not done; PBC, primary biliary cirrhosis.

Table 2 | Demographic, clinical and laboratory data in ICP patients and controls

\begin{tabular}{lcc} 
& $\mathrm{ICP}(\mathrm{n}=201)$ & Controls $(\mathrm{n}=198)$ \\
\hline Age (years) & $30(17-46)$ & $49(20-60)$ \\
Gender (women/men) & $201 / 0$ & $198 / 0$ \\
Liver cirrhosis (yes/no) & $0 / 201$ & $0 / 198$ \\
ALT (IU/I) & $112(4-1196)$ & $38(11-194)$ \\
AP (IU/I) & $312(58-1829)$ & $\mathrm{ND}$ \\
$\gamma$-GT (IU/I) & $30(5-473)$ & $34(12-1138)$ \\
Bilirubin (mg/dl) & $0.7(0.2-15.0)$ & $0.5(0.2-1.3)$
\end{tabular}

Values are given as medians (ranges), unless stated otherwise.

Abbreviations: see Table 1A. ICP, intrahepatic cholestasis of pregnancy.

p.148Ile conferred a significant risk $(\mathrm{OR}=3.94,95 \% \mathrm{CI}=0.91-$ $17.00, \mathrm{P}=0.048$ ) to require plasmapheresis (Figure 2, Table 3). On the other hand, this variant did not increase the risk of developing PBC per se (Supplementary Table 2), or cirrhosis in PBC patients (Supplementary Table 3), and was neither associated with laboratory

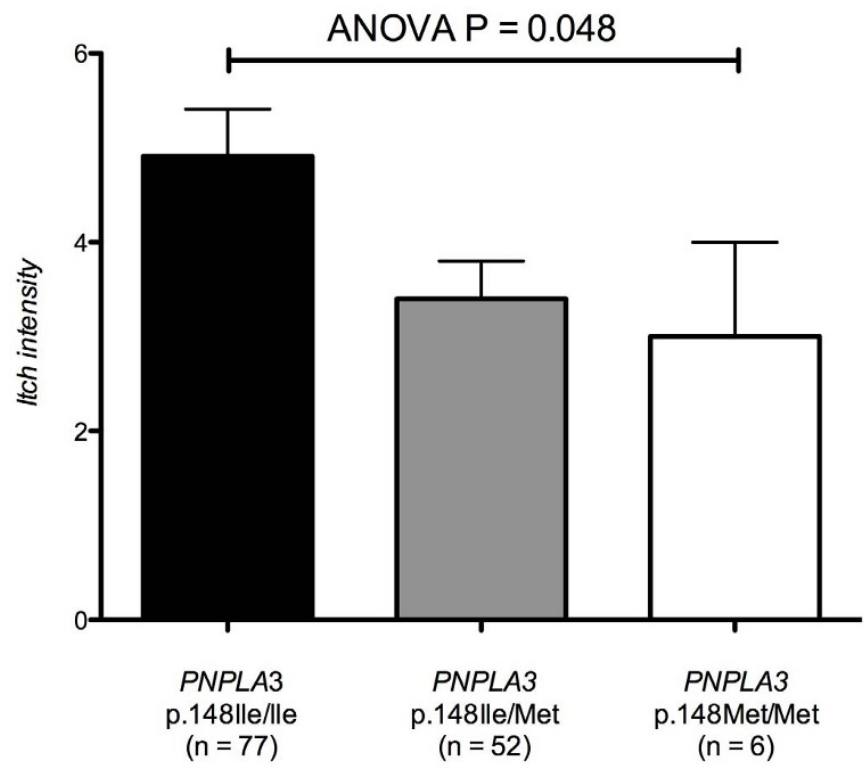

Figure $1 \mid$ Itch intensity in PBC patients stratified for PNPLA3 p.Ile148Met genotypes. Carriers of different PNPLA3 p.Ile148Met genotypes report significantly different intensity of pruritus (ANOVA $\mathrm{P}=0.048$ ), as quantified by the itch domain of the PBC- 40 questionnaire.

parameters of PBC (Supplementary Table 4) nor with domains of the PBC-40 questionnaires other than the intensity of pruritus (Supplementary Table 1).

Consistent with these observations, we detected a significantly increased frequency of the PNPLA3 allele p.148Ile in a second large independent cohort of patients with ICP who presented with itch during pregnancy as compared to controls (Figure 3, Supplementary Table 5). As illustrated in Supplementary Figure 1, this lead to a departure of the genotype distribution from Hardy-Weinberg equilibrium in ICP patients, supporting the genetic association. Patients

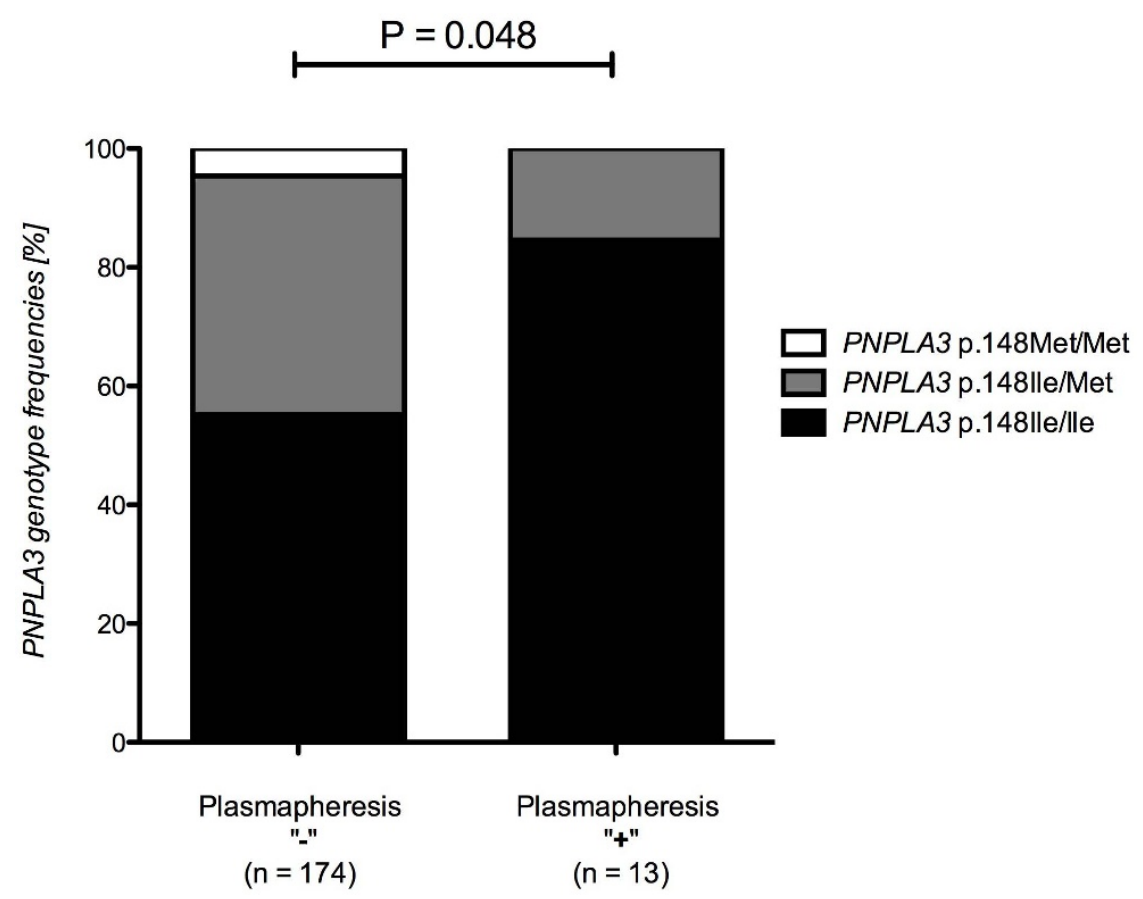

Figure $2 \mid$ PNPLA3 p.Ile148Met genotypes in PBC patients who did not and patients who required plasmapheresis for the treatment of pruritus. Patients who were treated with plasmapheresis carry the $P N P L A 3$ allele p.148Ile significantly (allelic 1 -df test $\mathrm{P}=0.048$ ) more often than patients who did not require this treatment. 
Table 3 | Distribution of PNPLA3 alleles and genotypes in PBC patients stratified according to the need for plasmapheresis to relieve pruritus

Count of alleles/genotypes

PNPLA3 p.lle 148Met alleles/genotypes

lle

Met

Ile/lle

$\mathrm{lle} /$ Met

Met/Met

Allelic 1-df test

[lle] $\leftrightarrow$ [Met]
Plasmapheresis $(+)(\mathrm{n}=13)$

24 (92.3)

$2(7.7)$

$11(84.6)$

$2(15.4)$

0 (0)

$\mathbf{P}$

0.048
Plasmapheresis $(-)(n=174)$

$262(76.3)$

$86(24.7)$

$96(55.2)$

$70(40.2)$

$8(4.6)$

OR $(95 \% \mathrm{CI})$

$3.94(0.91-17.00)$

Abbreviations: $\mathrm{Cl}$, confidence interval; lle, isoleucine; Met, methionine; OR, odds ratio; $\mathrm{p}$, protein (amino acid number); PBC, primary biliary cirrhosis; PNPLA3, adiponutrin.

carrying the PNPLA3 allele p.148Met were at significantly decreased risk $(\mathrm{OR}=0.66,95 \% \mathrm{CI}=0.47-0.92, \mathrm{P}=0.013)$ of presenting with ICP. Since pruritus is the major symptom at diagnosis of ICP, the lower frequency of the p.148Met allele in this cohort replicates the observation that this allele decreases the degree of cholestatic itch.

\section{Discussion}

This is the first study demonstrating an association of a genetic polymorphism with cholestatic pruritus. Here we report that the PNPLA3 allele p.148Met decreases itch severity in cholestatic patients. Our results are in line with functional analyses implicating that carriers of this allele display an increased metabolism of LPA ${ }^{7}$. We hypothesize that increased LPAAT $^{7}$ activities in liver and/or skin $^{10}$ of patients carrying the PNPLA3 allele p.148Met might be the mechanism that decreases itching, however further functional analyses are required. Moreover, our study identifies this PNPLA3 variant as potential genetic marker for therapy-refractory pruritus. Interestingly, our recently reported young female patient who developed severe refractory pruritus after acute hepatitis A infection as a result of hepatobiliary transporter variants ${ }^{12}$ is also a homozygous carrier of the susceptible PNPLA3 allele p.148Ile (M.K. and F.L., data on file).
The association between the PNPLA3 polymorphism and pruritus was independent from potential effects on liver function ${ }^{2-6,13}$ (Supplementary Tables 3 and 4). The lack of association of the PNPLA3 variant with liver cirrhosis in the $\mathrm{PBC}$ cohort indicates that the skin might be the major site where the antipruritic effects are exerted. Hence we conclude that the PNPLA3 variant p.Ile148Met is an example of biological pleiotropy ${ }^{14,15}$ with influence on more than one liver-related trait: In patients with chronic liver diseases the allele p.148Met is associated with disease progression ${ }^{16}$, but in case of cholestasis it might protect against itch. However, our results require further replication in additional cohorts. Since the PNPLA3 allele p.148Ile may to a certain extent explain the development of therapyrefractory pruritus, genotyping of the PNPLA3 variant p.Ile148Met might be included in the diagnostic work-up of patients with cholestatic liver conditions.

\section{Patients and Methods}

Patients with primary biliary cirrhosis (PBC). In total, we recruited 187 Polish PBC patients (age range 22-83 years, 166 females). All patients fulfilled the European Association for the Study of the Liver (EASL) criteria for the diagnosis of $\mathrm{PBC}^{8}$.

Table 1 presents the detailed description of this cohort. Liver function tests were determined by standard assays in fasted blood samples. In 135 patients, quality of life and intensity of itch were prospectively assessed with the PBC-40 questionnaire ${ }^{17}$.

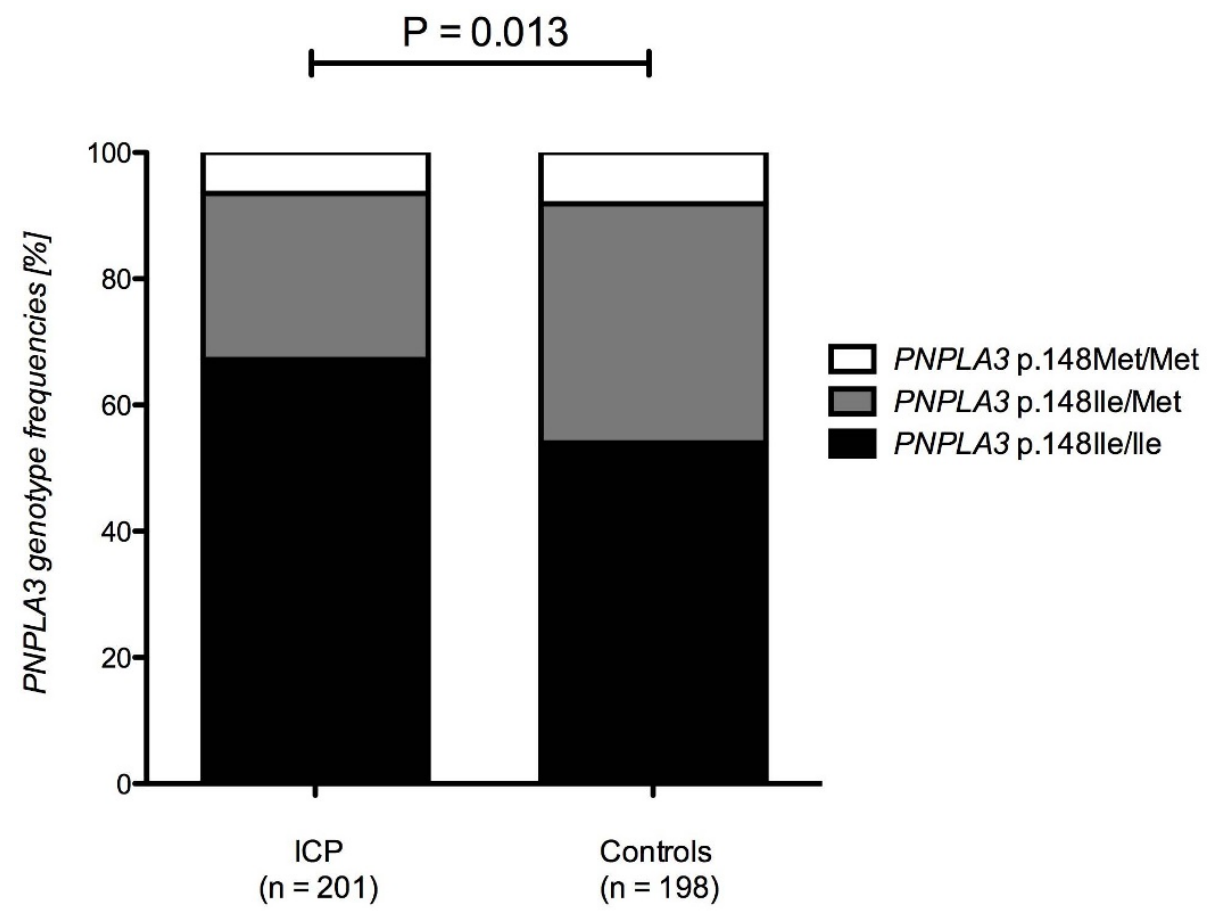

Figure $3 \mid$ PNPLA3 p.Ile148Met genotypes in ICP patients and sex-matched controls. Patients diagnosed with ICP present a significantly (allelic 1-df test $\mathrm{P}=0.013$ ) higher frequency of the PNPLA3 allele p.148Ile as compared to controls. 
Sixty-nine patients presented with histological, clinical and/or imaging features characteristic for liver cirrhosis. Among the PBC patients, a total of 13 who did not respond to pharmacological treatment of their pruritus (including ursodeoxycholic acid, colestyramine and rifampicin) were treated with plasmapheresis. The control cohort encompassed 250 healthy blood donors from the National Blood Services (Table 1$)^{18}$.

Patients with intrahepatic cholestasis of pregnancy (ICP). A cohort of 201 females with ICP (age range 17-46 years) was recruited between 2000 and 2013. Table 2 summarizes the details of this cohort. The included patients fulfilled the EASL criteria

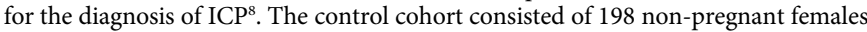
(age range 20-60 years) without any documented episodes of cholestasis during pregnancy. The study was conducted according to a study design approved by the local ethical committees, and informed consent was obtained from all study participants.

Genotyping. In all individuals, we genotyped the PNPLA3 variant p.Ile148Met (rs738409) as described ${ }^{6}$. Genomic DNA was isolated from EDTA anticoagulated blood using the membrane-based QIAamp DNA extraction protocol (Qiagen, Hilden, Germany).

Statistical analyses. The consistency of genotype frequencies with Hardy-Weinberg equilibrium (HWE) was tested using an exact test. Allele frequency differences were assessed by 1 -df chi $^{2}$ tests (http://ihg.gsf.de/cgi-bin/hw/hwal.pl). The study hypothesis was tested at a two-sided p-value of 0.05 . For further exploratory analyses, quantitative phenotypic data were analyzed using Mann-Whitney U or Fisher's protected least significant difference (PLSD) tests, and qualitative phenotypes were assessed in contingency tables.

1. Anstee, Q. M. \& Day, C. P. The genetics of NAFLD. Nat. Rev. Gastroenterol. Hepatol. 10, 645-655 (2013).

2. Romeo, S. et al. Genetic variation in PNPLA3 confers susceptibility to nonalcoholic fatty liver disease. Nat. Genet. 40, 1461-1465 (2008).

3. Valenti, L. et al. I148M patatin-like phospholipase domain-containing 3 gene variant and severity of pediatric nonalcoholic fatty liver disease. Hepatology. 52, 1274-1280 (2010)

4. Tian, C., Stokowski, R. P., Kershenobich, D., Ballinger, D. G. \& Hinds, D. A. Variant in PNPLA3 is associated with alcoholic liver disease. Nat. Genet. 42 21-23 (2010).

5. Stickel, F. et al. Genetic variation in the PNPLA3 gene is associated with alcoholic liver injury in Caucasians. Hepatology. 53, 86-95 (2011).

6. Krawczyk, M., Grünhage, F., Zimmer, V. \& Lammert, F. Variant adiponutrin (PNPLA3) represents a common fibrosis risk gene: non-invasive elastographybased study in chronic liver disease. J. Hepatol. 55, 299-306 (2011).

7. Kumari, M. et al. Adiponutrin functions as a nutritionally regulated lysophosphatidic acid acyltransferase. Cell Metab. 15, 691-702 (2012).

8. Beuers, U. et al. EASL Clinical Practice Guidelines: management of cholestatic liver diseases. J. Hepatol. 51, 237-267 (2009).

9. Karlsen, T. H. \& Boberg, K. M. Update on primary sclerosing cholangitis. J. Hepatol. 59, 571-582 (2013).

10. Huang, Y. et al. A feed-forward loop amplifies nutritional regulation of PNPLA3. Proc. Natl. Acad. Sci. USA. 107, 7892-7897 (2010).
11. Kremer, A. E. et al. Lysophosphatidic acid is a potential mediator of cholestatic pruritus. Gastroenterology. 139, 1008-1018 (2010).

12. Krawczyk, M., Grünhage, F., Langhirt, M., Bohle, R. M. \& Lammert, F. Prolonged cholestasis triggered by hepatitis A virus infection and variants of the hepatocanalicular phospholipid and bile salt transporters. Ann. Hepatol. 11, 710-714 (2012)

13. Krawczyk, M., Müllenbach, R., Weber, S. N., Zimmer, V. \& Lammert, F. Genome wide association studies and genetic risk assessment of liver diseases. Nat. Rev. Gastroenterol. Hepatol. 7, 669-681 (2010).

14. Solovieff, N., Cotsapas, C., Lee, P. H., Purcell, S. M. \& Smoller, J. W. Pleiotropy in complex traits: challenges and strategies. Nat. Rev. Genet. 14, 483-495 (2013).

15. Blair, D. R. et al. A nondegenerate code of deleterious variants in Mendelian loci contributes to complex disease risk. Cell. 155, 70-80 (2013).

16. Krawczyk, M., Portincasa, P. \& Lammert, F. PNPLA3-associated steatohepatitis: toward a gene-based classification of fatty liver disease. Semin. Liver. Dis. 33, 369-379 (2013).

17. Jacoby, A. et al. Development, validation, and evaluation of the PBC-40, a disease specific health related quality of life measure for primary biliary cirrhosis. Gut. 54, 1622-1629 (2005).

18. Kempinska-Podhorodecka, A. et al. TRAF1 gene polymorphism correlates with the titre of Gp210 antibody in patients with primary biliary cirrhosis. Clin. Dev. Immunol. 2012, 487521 (2012).

\section{Acknowledgments}

This work was supported by: National Science Centre, Poland (Grant 2011/02/NZ5/00321) and the HOMFOR program of Saarland University.

\section{Author contributions}

M.K., E.W., H.U.M., C.B., F.G., P.M. and F.L. recruited patients and controls for this study; M.M. performed the genotyping; M.K., M.M., P.M. and F.L. analyzed the data; M.K., P.M and F.L. drafted and edited the manuscript; P.M. and F.L. supervised the project and contributed equally to this study. M.K. had full access to all the data in the study and takes responsibility for the integrity of the data and the accuracy of the data analysis.

\section{Additional information}

Supplementary information accompanies this paper at http://www.nature.com/ scientificreports

Competing financial interests: The authors have no competing interests as defined by Nature Publishing Group, or other interests that might be perceived to influence the results and/or discussion reported in this paper.

How to cite this article: Krawczyk, M. et al. Variant adiponutrin confers genetic protection against cholestatic itch. Sci. Rep. 4, 6374; DOI:10.1038/srep06374 (2014).

This work is licensed under a Creative Commons Attribution 4.0 International License. The images or other third party material in this article are included in the article's Creative Commons license, unless indicated otherwise in the credit line; if the material is not included under the Creative Commons license, users will need to obtain permission from the license holder in order to reproduce the material. To view a copy of this license, visit http://creativecommons.org/licenses/by/4.0/ 